\title{
A Robust Adaptive Hydraulic Power Generation System for Jet Engines
}

\author{
G. Jacazio, A. Mornacchi, P. Ronco, and M. Sorli \\ Department of Mechanical and Aerospace Engineering, Politecnico di Torino, Italy \\ E-mail: giovanni.jacazio@polito.it, andrea.mornacchi@polito.it, pierantonio.ronco@polito.it, massimo.sorli@polito.it
}

\begin{abstract}
The paper presents an innovative hydraulic power generation system able to enhance performance, reliability and survivability of hydraulic systems used in military jet engines, as well as to allow a valuable power saving. This is obtained by a hydraulic power generation system architecture that uses variable pressure, smart control, emergency power source and suitable health management procedures. A key issue is to obtain all these functions while reducing to a minimum the number of additional components with respect to the conventional hydraulic power generation systems. The paper firstly presents the state-of-art of these systems and their critical issues, outlines the alternative solutions, and then describes architecture, characteristics and performance of the hydraulic power generation system that was eventually defined as a result of a research activity aimed at moving beyond the present state-of-art in this field.
\end{abstract}

Keywords: Hydraulic power generation, adaptive pressure, energy saving, jet engines, health management

\section{Problem statement}

Jet engines of several military aircraft are equipped with hydraulically powered actuation systems controlling the angular position of the stator blades of the high pressure compressor (VIGV = Variable Inlet Guide Vanes actuators) and actuation systems controlling the throat and exhaust areas of the nozzle (VEN = Variable Exhaust Nozzle actuators). Furthermore, the engines of some recent military aircraft are also capable of vectoring the thrust, which is typically obtained by individually controlling the positions of the VEN actuators placed around the engine nozzle. The nozzle actuators outputs are connected to a linkage mechanism which moves the nozzle petals such to orientate the flow of the exhaust gases, and hence the thrust, in the required direction.

The hydraulic power necessary for the operation of these actuators is obtained by a hydraulic power generation unit (HPGU) whose pump is driven by a shaft of the engine accessory gearbox, and an autonomous hydraulic circuit is thus created in the engine, which is separated from the aircraft hydraulic system. The engine hydraulic system uses a different oil, better suited for high temperature operation.

A few critical issues are associated to engine hydraulic systems and to their operation. Firstly, the system components operate in a severe temperature environment. The internal heat generation associated to the system operation, in combination with high temperatures of the surrounding air and of the components mounting points, creates a heavy thermal burden on the fuel cooled oil heat exchanger, with a resulting high temperature of the hydraulic fluid. This makes the system components (in particular the seals) more prone to damages, and reduces the bulk modulus of the hydraulic fluid with an ensuing worsening of the servoloops stability margins. Secondly, a loss of the hydraulic pressure leads to a loss of system operation. A loss of the system pressure may have several origins, but the most likely ones are by far: a pump failure, or a lack of hydraulic fluid due to a progressive depletion of the hydraulic reservoir resulting from external leakages. The engine design is normally such that in case of loss of system pressure, the VIGV and VEN actuators are driven by the aerodynamic loads to a fail-safe position preventing catastrophic consequences, but at the same time leading to a loss of engine thrust. This is a major, but still acceptable failure for a two-engine aircraft, but it becomes hazardous for a single-engine aircraft. A possible way of mitigating the loss of system pressure is to use the hydraulic power supply only for the VEN actuators, while using fuel as a working fluid for the VIGV actuators; this would leave the VIGV actuators operational in case of failure of the hydraulic pump, thereby allowing the engine to still be partially controlled and develop sufficient thrust. It is a viable solution which however brings about a heavier system due to the relatively low pressure of the fuel system and the resulting large size of the VIGV actuators.

A research activity was therefore conducted with the intent of mitigating the criticality of the issues outlined above. In particular, the main objectives of the research were to define a hydraulic power generation and actuation system for a jet engine able to provide control of variable inlet guide vanes, 
nozzle exhaust area and thrust orientation with the following characteristics:

- Use of high pressure hydraulic power source for all actuators

- Minimize the internal heat generation by making the system pressure adaptive to the actuators loads. It must be noticed that maximum loads occur for a very small fraction of the aircraft mission, thus unnecessary energy is generated and converted into heat by a constant pressure system

- Establish components characteristics and control laws such to ensure a dynamics of thrust vector control equivalent to that typical of the primary flight controls of a military aircraft

- Ensure a limited back-up power supply for allowing VIGV operation and controlled recentering of the nozzle actuators in case of loss of the system pump

- Implement prognostics and health management algorithms into the engine electronic control unit able to identify the precursors of critical system failures, thereby creating an alert before the failure develops

A hydraulic power generation and actuation system was hence defined to attain the above characteristics, and to do that while keeping the number of sensors to a minimum. The objective was not to add any sensor to those already present in the existing systems, and to take advantage of signals provided by sensors external to the hydraulic power generation system, but available in the engine electronic control unit. The system uses an engine driven variable delivery / variable pressure pump and an auxiliary, electrically driven, variable speed fixed displacement pump to assist the engine driven pump in ensuring the required frequency response for the actuators and as an emergency power source in case of failure of the main pump. In this latter case, a priority valve in the hydraulic distribution system favours the flow passage to the VIGV actuators that retain full performance while the nozzle actuators operate in a degraded mode. The system discharge pressure is established by an appropriate law embedded in the engine control electronics as a function of engine rating and nozzle actuators position; a pressure transducer located on the high pressure line provides the pressure signal necessary to close a pressure servoloop that operates by modulating the setting of the pressure compensator of the engine driven pump and the speed of the electrically driven pump. The modulation of the system pressure as a function of the actuators loads is a very effective way of reducing the heat generation, hence minimizing the power consumption and limiting the maximum temperature of the hydraulic fluid.

The signals provided by all system sensors (reservoir oil level quantity, temperature, filters differential pressure, pumps speeds, servovalves currents, actuators positions) are fused together and managed by appropriate algorithms in order to get a continuous indication of the system health and identify possible degradation patterns of its components.

All together, when compared with existing engine flow control systems, the one defined and thoroughly analyzed in the research activity looks more robust, less power demanding and better maintainable. The system concept, architecture, characteristics and performance are outlined in the proposed paper.

\section{State-of-Art}

Jet engines are required to operate at different conditions during the aircraft flight and some sort of control of the airflow must be provided in order to ensure an optimal engine performance over the entire range of operating conditions. This section of the paper briefly outlines the main features relevant to the airflow control.

\subsection{Variable inlet guide vanes}

Most of military jet engines are provided with an actuation system that controls the angular position of the stator vanes of the compressor. By doing so, the airflow leaving the stator vanes impinges the rotor vanes of the subsequent compressor stage with the most suitable angle of attack, thereby ensuring a good aerodynamic efficiency, averting the compressor stall and enabling a wider range of engine operating conditions. The compressor vanes provided with the capability of changing their orientation are known as variable inlet guide vanes (VIGV). The stator vanes are actually equipped with levers rotating about a fixed hinge and connected at one end to a unison ring placed around the compressor [1]. By modulating the position of the unison ring, the stator vanes levers are rotated and the vane angle is hence varied.

\subsection{Variable exhaust nozzle}

A second feature used in several military jet engines to optimize the airflow is the variation of the nozzle flow area (VEN = Variable Exhaust Nozzle). The nozzle is the engine component where the gas jet accelerates from moderate speed in the turbine to the higher velocity of ejection from the engine to generate the thrust. A variable exhaust nozzle requires an actuation system to control the position of a mechanical element driving a series of linkages connected to the nozzle petals. The variation of the actuators output position eventually causes the variation of the nozzle area,

\subsection{Thrust vectoring}

In addition to variable geometry, some modern military jet engines are provided with thrust vectoring capability (TVN $=$ Thrust Vectoring Nozzle), which allows to vary the direction of the thrust, thereby enhancing the aircraft maneuverability. Thrust vectoring technology has been successfully demonstrated on several programs to provide tactical maneuvering advantages in the very slow speed, very high angle-of-attack flight regime. This technology has matured to the extent that it is being incorporated into present day fighter aircraft designs [2-7].

\subsection{Actuation systems}

To accomplish VIGV, VEN and TVN functions actuation systems are required to control the position of the inlet guide vanes unison ring and of the nozzle petals such to obtain the 
desired guide vanes angle of attack, nozzle area and thrust direction. Three different actuation technologies can in principle be used:

- Hydraulics: A dedicated hydraulic power generation unit with an engine driven pump provides high pressure hydraulic power supply to the actuators

- Fueldraulics: The actuators are supplied by pressurized fuel drawn from the engine fuel system

- Electrical: Electromechanical actuators accept the controlled electrical power supply from the engine electrical system

Aircraft engines that are provided only with VIGVs, but do not have nozzle variable exhaust area control typically use fueldraulic actuators because the loads are small and the size of the actuators is acceptable and can be accommodate in the engine envelope also if the fuel system pressure as low as 6-7 MPa.

Studies have been recently conducted on VIGVs controlled by elecromechanical actuators [8] within the research activities on a more electric engine, but no application has so far followed. When nozzle control is required, fueldraulics is not any longer a viable technology because the low pressure of the engine fuel system would lead to an unacceptable large size of the actuators.

Electromechanical actuation technology, though appealing for several reasons, still falls short of meeting the envelope and safety requirements for the nozzle actuators performing either VEN or combined VEN + TVN functions. The critical issues of electromechanical actuation for these applications are the envelope constraints in the nozzle area, the thermal management and the possibility of actuators seizure, that could lead to a hazardous condition for a single engine aircraft.

For these reasons, hydraulic actuation is the only technology presently used in engines with VEN, or VEN+TVN capability. The research activity presented in this paper was focused to engines featuring a VEN, or a VEN+TVN in addition to the VIGVs, thus hydraulic actuation was the technology being considered.

\subsection{Hydraulic power generation}

Different architectures of hydraulic power generation systems can be considered for providing the hydraulic power supply to the engine actuators. Firstly, the overall hydraulic actuation system architecture can be based on a valve controlled system in which the actuators flows are controlled by throttling the flows by means of servovalves, or on a pump controlled system in which individual overcenter variable delivery pumps interface with the corresponding actuators. Although this second concept has the merit of automatically adapting the pumps delivery pressures to the actuators loads, it leads to a too heavy and expensive system because of the large number of pumps; moreover, the continuous tare flow of the servovalves controlling the variable delivery mechanisms of the pumps reduces the overall energy saving achievable from the pump control concept.
The solution typically used for the hydraulic power generation units (HPGU) of engine hydraulic systems is thus to have a pressure compensated, variable delivery pump providing the constant pressure variable flow required to move the actuators, plus the flow necessary to compensate the internal leakages. These HPGUs are sized on the basis of the maximum actuators speed and load determining the maximum flow and pressure to be provided by the HPGU. This sizing ensures that the actuators performance is attained, but does not optimize the energy consumption. The combination of actuators maximum speed and load typically occurs for a very limited portion of the aircraft flight envelope and may last only a few percent of the total aircraft flight time. When loads lower than maximum act upon the actuators, energy is lost through the control valves and heat is generated. Heat is rejected from the engine hydraulic system to a fuel cooled heat exchanger, but there are flight conditions in which the fuel entering into the heat exchanger is already at high temperature, which thwarts the cooling of the hydraulic fluid. Minimizing the heat generation is therefore paramount for the engine hydraulic systems.

\subsection{Existing solution for heat load reduction}

A load sensing hydraulic system seems the logical solution for reducing the heat generation. These systems have been extensively used in industrial and earth moving machinery applications, but are less suitable for the requirements typical of the jet engine hydraulic systems that supply servoactuators requiring a high frequency response. A viable solution for reducing the heat generation of a jet engine hydraulic system was however developed for the air flow control system installed on a reheated turbofan engine for a front-line fighter aircraft and is described in [9]. An analysis of this system is presented in [10]. This hydraulic system supplies the VIGV and the VEN actuators, and uses a variable delivery / variable pressure pump in which a modified pump pressure compensator changes the compensation pressure as a function of the load acting upon the VEN actuators, which are mechanically synchronized and controlled by a single servovalve.

The flow required for the VEN actuators operation is an order of magnitude greater than that required for the VIGV operation; therefore, this hydraulic system was designed to make the pump delivery pressure dependent only on the VEN actuators load. The pump delivery pressure varies in a well defined range depending on the VEN actuators loads and the VIGV actuators were sized to be capable of providing full performance also for the minimum pressure condition. This architecture of the hydraulic system has the merit of allowing a great reduction of heat generation with a very simple design; it leads to a slight greater size of the VIGV actuators, which is however acceptable due to their low absolute dimensions. When all internal losses of the hydraulic system are accounted for, including the pump frictional losses, the heat generation of a variable delivery / variable pressure system for a typical flight mission is well below that obtained from a conventional constant pressure system. 
This variable delivery / variable pressure hydraulic system is therefore a good solution for a reheated turbofan engine equipped with VEN and VIGV actuators, and when the aircraft is provided with two engines. For the case of a single engine aircraft this system becomes critical since the loss of the hydraulic power supply resulting from a pump failure would lead to the inability of controlling the VIGVs, which could result in an engine shutdown and thus to a flight safety critical condition.

Moreover, this same hydraulic system is good for an air flow control system in which four VEN actuators are mechanically synchronized and controlled by a single servovalve, but it falls short of optimizing the delivery pressure for engines with TVN capability, in which four servoactuators are independently controlled by four servovalves.

Devising a new architecture for an engine hydraulic system suitable for single engine operation and for TVN control, which is also minimizing the heat generation was hence the objective of the research activity described in this paper.

\section{Smart adaptive system architecture}

\subsection{Objectives}

While defining the new HPGU architecture, the following main objectives were addressed:

- The engine driven pump is the component with the highest failure rate in the existing systems

- Minimizing the heat generation is paramount

- It is desirable that TVN control meets the dynamic response requirements of primary flight controls in order to enhance the aircraft maneuverability and also provide a backup in case of primary flight controls failure

- Control of the VIGV has priority over VEN/TVN control because loss of VIGV control could lead to a loss of engine thrust and to a flight safety critical condition for a single engine aircraft

- Probability of loss of hydraulic power supply to either VIGV or VEN/TVN servoactuators must be lower than $1 \times 10^{-7}$ per engine operating hour, whilst probability of total loss of operation of VIGVs must be lower than $1 \times 10^{-9}$ per engine operating hour

- Maintainability improvement over existing systems is desired

- HPGU operation is controlled and monitored by a dual Digital Engine Control Unit (DECU)

The new HPGU architecture was hence defined such to meet the above objectives

\subsection{HPGU architecture}

The architecture of the HPGU is shown in the diagram of Fig. 1. The overall system is single hydraulic with dual electrical control, but an electrical backup actuation is provided to the VIGVs to ensure that the flight safety target is met.

The hydraulic fluid is stored in a bootstrap type selfpressurizing reservoir, consisting of a differential area piston in which the high pressure delivered by the pumps is provided to the low area side of the piston. The resulting force is balanced by an equal force acting in the opposite direction on the large area side of the piston, which determines the value of the pressure of the hydraulic fluid stored in the reservoir. With a ratio of 40 between the large and low areas of the differential piston, a reservoir pressure of $0.5 \mathrm{MPa}$ is obtained for a delivery pressure of $20 \mathrm{MPa}$. That reservoir pressure is sufficient to avoid cavitation at the pumps suction for any operating condition, including start up at very low temperature. The reservoir accepts the return fluid from the hydraulic system and has an additional port for connection to an overfill/overtemperature relief valve that opens in case of inadvertent overfilling the hydraulic circuit, or of excessive volumetric expansion of the hydraulic fluid caused by abnormal high temperature resulting from HPGU internal failures. Opening of the relief valve prevents a fracture of the reservoir with the loss of the hydraulic system. It must be noticed that when the system is filled up on ground with the hydraulic fluid the plug placed soon downstream the overfill/overtemperature relief valve is removed and the corresponding port is connected to the reservoir of the ground support hydraulic unit. Therefore, if the reservoir is inadvertently overfilled and the reservoir piston is forced against its mechanical stops, the relief valve opens and the hydraulic fluid is returned back to the external reservoir, thereby preventing any external spillage. When the hydraulic system is operating, if an abnormal volumetric expansion forces the reservoir piston against its mechanical stops and the relief valve opens, the plug is now in place and the pressure increase causes a safety disc to fracture and the hydraulic fluid is vented overboard. After the excess volume has been vented, the pressure in the reservoir is reduced and the relief valve closes preventing any further passage of the hydraulic fluid and thus a depletion of the hydraulic system. Operation can then continue unabated. A visual indicator placed in the overboard vent line allows the maintenance crew to detect that hydraulic fluid spillage has occurred in flight and take the necessary maintenance actions.

The reservoir is provided with a hydraulic fluid level sensor, a low hydraulic fluid level switch and a temperature sensor. The signals provided by these sensors and switches are used by the health usage and monitoring system outlined at the end of the paper.

Hydraulic pressure is generated by two pumps: an engine driven pump and an electrically driven pump. The flows delivered by the two pumps are eventually summed upstream of the high pressure filter unit. 


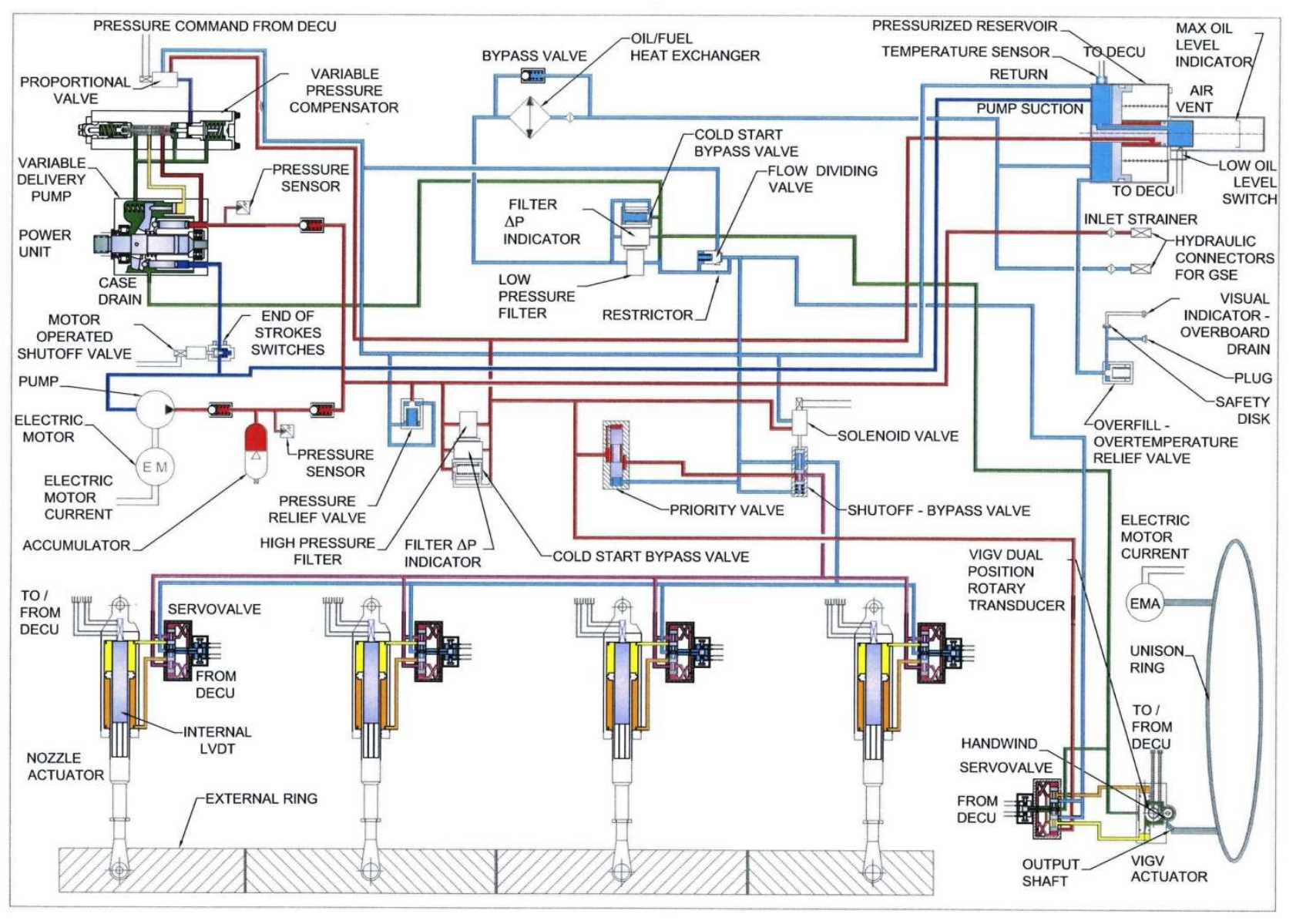

Figure 1: System block diagram

The engine driven pump is the primary pump of the hydraulic system; it is an axial piston variable delivery, pressure compensated pump mechanically connected to a shaft of the engine accessory gearbox. An important feature of the engine driven pump is that it uses a modified pressure compensator able to change the compensation pressure according to the actuators loads. The variable pressure compensator is a modified version of the conventional pressure compensator. A pilot pressure signal acts on an auxiliary piston which on its turn exerts a force on the compensator spool that is a function of the pilot pressure. The end result is to change the preload of the pressure compensator spring, thereby making the pump discharge pressure a function of the pilot pressure.

A pressure transducer located on the pump discharge line measures the pump discharge pressure and provides this information to the DECU. The pump compensator pilot pressure is generated by a 3-way pressure control valve accepting the electrical command from the DECU. This arrangement enables the DECU to vary the pump discharge pressure according to the actuation system needs as it will described in the following section 4. A check valve downstream of the pressure transducer prevents a reverse flow from the hydraulic system to the engine driven pump.

The electric driven pump acts as an auxiliary unit for the hydraulic system; it is an axial piston, fixed displacement pump driven by a speed controlled brushless dc electric motor. A gas charged accumulator is placed on the delivery line of the electric driven pump; the delivery line downstream of the accumulator is then connected to the delivery line of the engine driven pump upstream of the high pressure filter unit. A check valve prevents a reverse flow from the hydraulic system back to the accumulator. A pressure transducer measures the accumulator pressure and provides this information to the DECU that eventually controls the electric motor speed to maintain the accumulator pressure at the required value.

The control of the hydraulic fluid cleanliness is performed by high pressure and low pressure filter units. The high pressure filter unit consists of a filter element with a $\beta_{200}=3$ $\mu \mathrm{m}$ and a differential pressure transducer. The high filtering grade is recommended to protect the actuators servovalves and ensure their correct operation over the entire system life. The differential pressure transducer provides indication of the pressure drop through the filter enabling the DECU to monitor the HPGU health as it will be outlined in section 5 . No bypass valve is used for the high pressure filter unit because of the high sensitivity of the actuators servovalves to contamination. If the filter creates a large pressure drop, also if temporary, it is preferable to accept a reduction of the actuation systems performance rather than risk to contaminate the actuators servovalves. 
The low pressure filter unit consists of a flow dividing valve, a filter element with a $\beta_{200}=10 \mu \mathrm{m}$, a differential pressure transducer and a bypass valve. The flow dividing valve consists of a spring loaded pressure relief valve and a restrictor placed in parallel. The flow dividing valve accepts the return flow from the actuators at its inlet and divides it into two output flows. If the inlet flow is small, as it is for most of the operating time, the flow passes entirely through the restrictor because the pressure drop created by the passage of this flow through the restrictor is lower than required to open the relief valve. If the actuators are commanded to move such to create a large flow, the pressure drop through the restrictor exceeds the limit of the relief valve that then opens bypassing the excess flow directly to the oil/fuel heat exchanger. In this case, part of the hydraulic fluid is not filtered by the low pressure filter, but this is not detrimental for the hydraulic system operation. The purpose of the low pressure filter is in fact to protect the pump from excessive wear, which is a progressive process. The passage of a limited quantity of unfiltered hydraulic fluid from the return line to the reservoir and eventually to the pump suction does not harm the pump operation, nor contribute significantly to the pump wear since the contaminants entrained by this flow will be eventually captured by either the high or low pressure filters as the fluid continues to circulate through the hydraulic system. Moreover, the contamination level of the flow returning from the actuators is normally low, and it is thus acceptable that occasional return flow spikes are not filtered. On the contrary, the drain flows from the two pumps are routed directly at the return filter inlet since they carry most of the contaminants generated by the wear of the pumps moving parts.

A differential pressure transducer senses the pressure drop through the filter element and provides the relevant signal to the DECU for monitoring the HPGU health status. A bypass valve is placed in parallel to the filter element to prevent an excessive backpressure in the return line in case of actuators operation during a very cold start, or of filter element clogging. An excessive backpressure in the return line could lead to a damage of the return line components of the hydraulic system, including the servovalves return paths. Opening of the low pressure filter bypass valve is signalled by a visual indicator to alert the maintenance crew; the visual indicator is provided with a thermal lockout to avoid its activation at low temperature since opening of the bypass valve in that condition is not an abnormal behaviour.

The above described configuration of the low pressure filter unit offers the advantage of ensuring an efficient control of the hydraulic fluid cleanliness with a low size filter element, which entails lower HPGU size, weight and reduced maintenance costs. The mean flow through the hydraulic system during a flight is typically lower than $10 \%$ of the maximum flow, hence a much greater filter would be required if designed to accept the rated system flow.

The total return flow leaving the low pressure filter unit passes through a fuel cooled oil heat exchanger which is also provided with a bypass valve to prevent excessive pressure drop in case of system operation during a cold start-up. It must be noticed that the fuel used as a coolant for the hydraulic fluid of engine hydraulic system has already been used to cool other aircraft system and may enter into the heat exchanger at a relatively high temperature; this makes highly important to minimize the energy losses in the engine hydraulic system to limit insofar as possible the maximum temperature of the hydraulic fluid.

A system pressure relief valve is placed upstream of the high pressure filter unit with the purpose of protecting the hydraulic system from abnormal pressures that could arise as a result of abnormal transients of the actuation systems, or of a failure of any of the two pumps. Section 5 will describe the recovery actions in case of a pump failure.

A motor operated shutoff valve is placed on the suction line of the engine driven pump. When open, this valve has a large enough passage to create minimum pressure drop, thereby avoiding any risk of cavitation at the pump suction. The purpose of this valve is to isolate the engine driven pump in case of its failure. The shutoff valve is provided with end-of-travel switches to allow a periodical check of its operation, thereby avoiding dormant failures.

The clean high pressure fluid leaving the high pressure filter is directed to a priority valve whose purpose is to ensure that the VIGV servoactuator always has the hydraulic supply fluid necessary for its operation. The priority valve is a 3way valve accepting the hydraulic fluid from the high pressure line and directing it toward the VIGV and the VEN/TVN actuators. The hydraulic system is sized such that under normal conditions both VIGV and VEN/TVN actuators receive all necessary flow for their operation. However, in case of reduced flow delivery, the priority valve progressively closes the passage of the VEN/TVN actuators line, hence throttling the flow to these actuators while leaving the VIGV flow unabated. This function is obtained by sensing the pressure in the hydraulic line connected to the VIGV servoactuator pressure supply. If this pressure decreases below $20 \mathrm{MPa}$, the priority valve spool moves in the direction of closing the port of the hydraulic line connected to the VEN/TVN actuators pressure supply.

A solenoid operated shutoff/bypass valve is placed on the supply line to the VEN/TVN actuators which consist of servovalve operated linear hydraulic actuators, each provided with a position transducer. The purpose of this valve is to cut off the hydraulic power supply to these actuators in case of a failure, thereby inhibiting their operation and isolating the VEN/TVN part of the entire system from the hydraulic power supply. At the same time, the supply and return lines downstream of the shutoff/bypass valve are interconnected, which allows the aerodynamic loads acting on the nozzle to bring back the actuators to an aligned, fail safe position..

The HPGU has two hydraulic fittings for connection to an external ground support equipment which is used to fill the system with the hydraulic fluid and circulate it through system passageways, pipes and components to ensure a complete air removal. 


\subsection{Nozzle servoactuators}

Four linear hydraulic servoactuators placed around the engine nozzle are used to perform VEN and TVN control. Each of the four servoactuator is comprised of a slightly unbalanced area linear hydraulic actuator whose flow is controlled by an electrohydraulic servovalve. A small bypass orifice placed across the servovalve control ports allows the actuator recentering under the external pressure loads in case of loss of hydraulic pressure, and helps the position servoloop stability. The two servovalve coils are independent and accept the control current from the two separate sections of the DECU. A dual electrical position transducer placed inside the piston rod provides a dual redundant position indication to the DECU, which is used both to close the position servoloop and to monitor the system operation.

The unbalanced area configuration for the nozzle actuators entails more reservoir volume, but on the other hand it greatly reduces the actuators length and allows the installation of the position transducer inside the actuator, which is beneficial for the transducer because of the hot environment around the engine nozzle.

\subsection{VIGVs servoactuators}

It was stated in paragraph 3.1 that one of the system objectives is to keep the probability of total loss of VIGVs operation lower than $1 \times 10^{-9}$ per engine operating hour. This will allow the applicability of this system to a single-engine aircraft. Although the HPGU architecture is based on two pumps, meeting the $1 \times 10^{-9}$ probability of total loss of the VIGVs operation is not possible unless the two VIGV actuators are connected to two separate HPGUs. A possibility could be to connect one VIGV actuator and the VEN/TVN actuators to the main HPGU, and the other VIGV actuator two an auxiliary HPGU. Although this second HPGU could be miniaturized, still its weight, envelope and cost would be unacceptable, because it would anyhow be a complex unit made up by several components to simply provide the hydraulic power supply to a small servoactuator using a limited amount of flow.

The most logical solution is to have a VIGV actuator connected to the HPGU acting as a master actuator, and a second VIGV actuator consisting of a fueldraulic, or of an electromechanical actuator, acting as a backup actuator. The author preferred choice for the backup actuator is for an electromechanical actuator because it will eventually lead to lower size and weight. In case of failure of the master actuator, it is necessary to control the position of the VIGVs, and the actuator must be able to develop the full load, but the actuation rate could be reduced. There are engine operating conditions in which the fuel pressure is low, which requires a large area of a fueldraulic actuator to balance the VIGV load. On the contrary, an electromechanical actuator can be designed as a geared system in which a small motor torque is amplified by a compact gear reducer, while speed is reduced. An optimum compromise can eventually be reached between motor size, gear ratio and output speed such to make the actuator able to operate against the maximum load at a reduced, albeit still acceptable, rate.

The solution eventually considered in this research activity was hence to have a hydraulic master actuator and an electromechanical backup actuator. The hydraulic master actuator is a servovalve controlled actuator with a rotary output and a dual electrical rotary position transducer. The servovalve has configuration and operational characteristics identical to those of the nozzle actuators servovalves, and a small bypass orifice is also placed across the servovalve control ports.

The electromechanical actuator consists of a brushless dc motor with its electric motor drive and a gear reducer. A resolver on the motor shaft provides rotor position information to the electric motor drive to perform the appropriate switching of the motor currents and close a motor speed loop. An absolute dual electrical position sensor provides position indication to close the position servoloop. The electric motor power supply is automatically switched to the active electrical lane.

\subsection{System architecture summary}

The architecture of the entire system described in the above paragraphs is then:

- Single hydraulic HPGU with dual pressure supply generation and pressure adaptiveness to the actuators loads

- Nozzle servoactuators consisting of servovalve controlled linear hydraulic actuators, each provided with an internal position transducer for VEN/TVN control

- Master and auxiliary VIGV servoactuators for VIGV position control. The master servoactuator is a servovalve controlled hydraulic actuator with a rotary output, the auxiliary servoactuator is an electromechanical actuator. The actuators are provided with a rotary position transducer

- System control is performed by a DECU consisting of two independent, mutually isolated, lanes operating in an active-standby mode

- All sensors and switches are dual electrical interfacing with the two DECU lanes

- The electrical power supply to the electric motors of auxiliary pump, engine pump suction shutoff valve and auxiliary VIGV servoactuator is automatically switched to the electrical supply of the active DECU lane

\section{System operation}

\subsection{Hydraulic power generation}

Under normal operating conditions the hydraulic power is simultaneously provided by the engine driven and by the electric driven pumps. The engine driven pump is a variable delivery unit whose input shaft speed depends on the engine speed and is not a controlled variable. The pump is hence designed with a maximum displacement such to provide the maximum flow also for the minimum speed condition that occurs when the engine is in ground idle. 
The electric driven pump is a fixed displacement unit whose shaft speed is controlled by regulating the electric motor speed. The pump is sized to provide the flow necessary for full rate operation of the VIGV servoactuator and for $10 \%$ rate of the VEN/TVN servoactuators.

The hydraulic fluid side of the gas charged accumulator is connected to the portion of the electric pump discharge line between two check valves. In this way, the liquid side of the accumulator can be filled only by the hydraulic fluid delivered by the electric motor pump and not by the engine driven pump. This is instrumental in allowing a good dynamic operation of the VEN/TVN servoactuators under certain operating conditions as it will be described later in this section. The accumulator can then provide pressurized fluid flow to the system pressure line when this pressure falls below the pressure prevailing in the accumulator.

\subsection{System pressure control}

One of the key features of the HPGU is the adaptation of the system pressure to the loads acting on the VEN/TVN actuators. The loads acting on these actuators are not related to the loads acting on the VIGV master actuator; therefore, this actuator is sized to develop the full load when the system pressure is at the minimum of its variation range. A tradeoff analysis led to the conclusion to set the variation range of the system supply pressure from a minimum of 21 $\mathrm{MPa}$ to a maximum of $35 \mathrm{MPa}$. Pressures lower than 21 $\mathrm{MPa}$ would have led to a too large VIGV master actuator, while the upper limit of $35 \mathrm{MPa}$ is the present state-of-art for aircraft hydraulic systems.

The value of the required system supply pressure is not determined by measuring the loads acting on the VEN/TVN actuators, or by measuring the pressure differentials across the control lines of those actuators. That solution is in principle possible, but would have led to a very complex system, with a large number of components and a large number of wires, with added weight and reduced reliability. The solution devised for this system is to implement an engine model in the DECU that computes the values of the loads acing on the VEN/TVN actuators based on the engine conditions. In fact, the DECU has all the necessary information to perform that calculation. It has available the engine rating, the aircraft speed and altitude, the fuel flow rate as well as command and position of all actuators. An appropriate algorithm can thus perform real time computation of the actuators loads and hence generate the value of the optimum system supply pressure for that operating condition. A similar concept is proposed for the hydraulic system of high-speed tilting trains [11].

When the loads on the VEN/TVN actuators are below a limit, the system pressure is kept constant at $21 \mathrm{MPa}$; this pressure is sufficient to ensure the full performance VIGV operation up to the maximum load and for full performance VEN/TVN operation up to the limit load, which normally occurs for more than $90 \%$ of the flight time. When the VEN/TVN loads grow higher than the limit, the DECU issues an appropriate signal to increase the system pressure as necessary. This is accomplished by creating two pressure control loops: one for the engine driven pump and one for the electric driven pump.
The pressure control loop of the engine driven pump uses as a feedback signal the pressure transducer of that pump discharge line; the pressure error is processed by a proportional control law to generate the electrical input signal to the pressure control valve modulating the pilot pressure for the pump pressure compensator. As a result, the pressure compensator setting is changed and the engine pump discharge pressure is accordingly modified.

A pressure control loop is also created to control the discharge pressure of the electric driven pump. Again, the pressure transducer of that pump discharge line provides the feedback signal to close the pressure servoloop and generate the pressure error which is processed by a proportional control law. The resulting control signal is accepted as a speed signal by the pump electric motor drive; the pump speed is hence adjusted such to maintain the discharge pressure at the required value.

Since the output flows of the two pumps are eventually merged, a concern is the possible insurgence of a hunting effect between the two parallel pressure control loops. To avoid that, the pressure command for the engine driven pump is set by the DECU to a value slightly greater than that for the electric driven pump. For the prevailing load conditions the pressure command for the engine driven pump is then set at $21 \mathrm{MPa}$, while that for the electric driven pump is set at $20.5 \mathrm{MPa}$. Therefore, in these conditions the main function of the electric driven pump is to keep the accumulator pressurized at $20.5 \mathrm{MPa}$, while the actuators flow is provided by the engine driven pump. However, should the system pressure temporarily fall below $20.5 \mathrm{MPa}$, the accumulator will deliver the pressurized fluid volume necessary to maintain the system pressure at $20.5 \mathrm{MPa}$ and ensure the actuators dynamic response. It must in fact be noted that the time required by the variable delivery mechanism of the engine driven pump to go from zero to full displacement could be 30 to 40 milliseconds. Without the reserve fluid provided by the accumulator, this would result in some initial delay of the VEN/TVN actuators response following a large step command, while the presence of the accumulator ensures a fast dynamic response.

If conditions are detected by the DECU requiring a system pressure above the $21 \mathrm{MPa}$ base threshold, the pressure command computed by the DECU algorithms is provided to the two pressure control loops. As it was mentioned before, the flow delivered by the engine driven pump cannot be directed to the accumulator, that can only be replenished by the flow delivered by the electric driven pump. The reason for this solution is that if no check valve were present downstream of the accumulator, if a command is issued by the DECU to the VEN/TVN servoactuators simultaneously to the variation of the pressure set, the actuators response could suffer from a large delay because a large portion of the engine driven pump flow would be drawn to replenish the accumulator. It is therefore much better to allow all the engine driven pump flow to be available to the actuators while leaving to the electric driven pump the task of providing the amount of fluid volume necessary to bring the accumulator pressure to the required value. Of course, the 
VEN/TVN actuators dynamic response for this particular operating condition might fall short of entirely meeting the requirements because of the time response of the variable delivery mechanism of the engine driven pump. However, this shortfall is negligible in most of the cases, and has some effect only when a very large step command is given to the nozzle actuators either for VEN or TVN operation.

Figure 3 shows the most critical condition of a nozzle initially aligned along the engine axis, which receives a full deflection command for which the actuators will be subjected to the maximum load and hence to the maximum pressure. When the DECU receives from the aircraft flight control system the new position demand, it converts it into a position command varying in time with a rate equal to the maximum design speed of the actuators and it simultaneously issues the new pressure command. The nozzle position command $x_{\text {setN }}$ and response $x_{N}$ for this severe condition, as well as the variation of the actuators and of the supply pressure are shown in Fig. 2. The actuator response has an initial maximum transient delay of about $100 \mathrm{~ms}$ due to the combined time delay of the supply pressure control system and variable delivery mechanism of the pump; the delay of the actuator response progressively reduces as the pump reacts. The $100 \mathrm{~ms}$ initial delay is large, but it is still acceptable for this type of command.

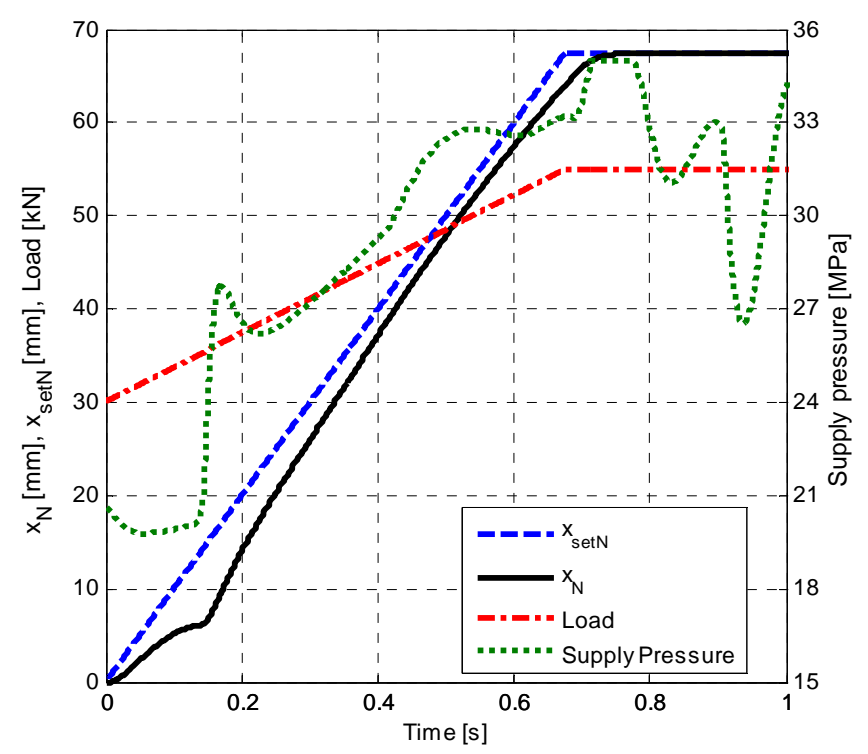

Figure 2: TVN transient following a full deflection command with associated actuators load increase from minimum to maximum

\section{Performance}

\subsection{Mathematical model}

The system performance was assessed for all range of operating conditions with the help of a dedicated mathematical model representative of the entire air flow control system. PI control laws with a small dead band and a saturation on the integrator path were used for VIGV and VEN/TVN position servoloops, while a simple proportional control was used for the pressure control loops.
The mathematical model was a "physical type" model and was implemented as a Matlab/Simulink software code. The model is of non-linear type and provides the values of the all state variables versus time. The independent variables are:

- Nozzle actuators position commands

- Variable inlet guide vanes actuators position command

- $\quad$ Nozzle and VIGV actuators loads

- $\quad$ Engine rating (pump speed)

The model accepts the oil temperature as a parameter and will thus compute the relevant characteristics of the resistance and capacitance of the hydraulic components. The model is essentially organized in different blocks. A master block reads the input variables and data, and then manages the simulation. At definite intervals of the simulation time the master block prints the value of all the main variables and stores most of them in a file for subsequent plotting. The other blocks are each dedicated to one of the subsystems and interface with the master block. A very detailed model was of each of the subsystem components was developed in order to obtain an accurate simulation. An example of the simulation accuracy reached by mathematical models of hydraulic components is presented in [12]. The DECU characteristics such as A/D converter resolution, recursion rate, computation time were duly addressed by the mathematical model.

\subsection{Dynamic response}

The results of the simulations showed the ability of the system to meet the time and frequency response requirements typical for a primary flight control of a front line aircraft. As an example, Fig. 3 shows the time response to a small step command to the nozzle actuators in VEN mode equal to $3 \%$ of the full actuators travel. The time required to reach the commanded position is equal to $25 \mathrm{~ms}$ in agreement with the specifications.

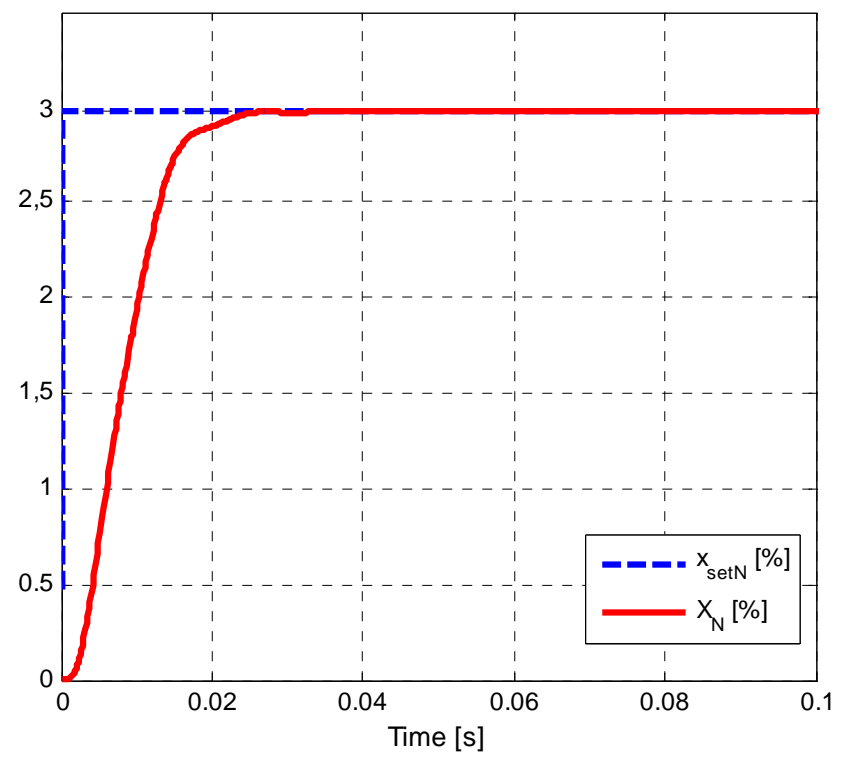

Figure 3: VEN transient for a small step position command

Figure 4 shows the time response to a large step command, equal to $30 \%$ of the full actuators travel. The time required 
to reach the commanded position is in this case equal to 160 $\mathrm{ms}$, which shows the ability of the nozzle actuators to operate very fast.

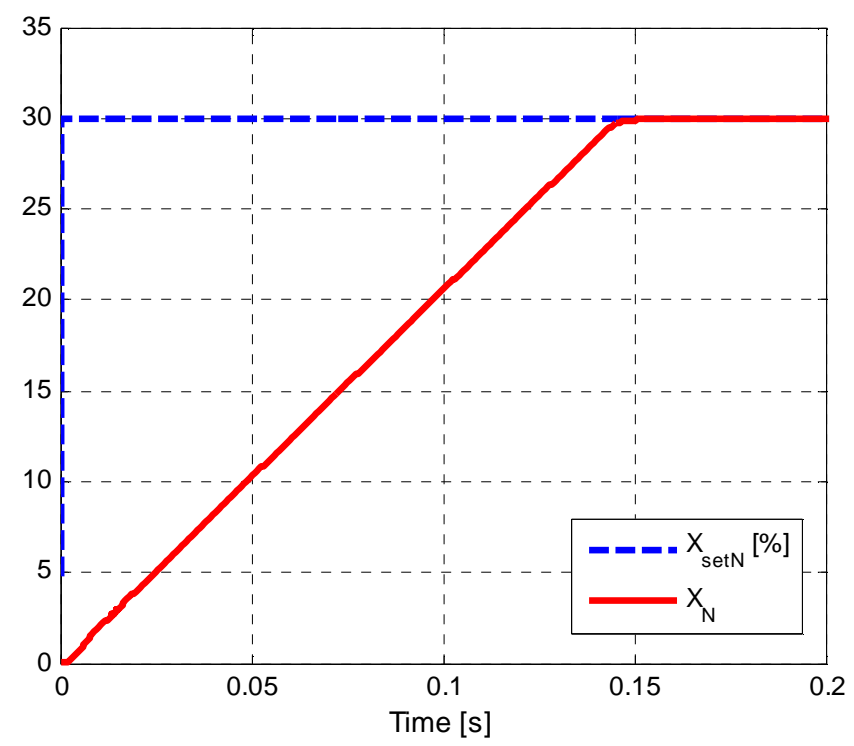

Figure 4: VEN transient for a large step position command

The following Fig. 5 shows the nozzle servoactuators frequency response when commanded in TVN mode. As it can be seen from the figure, the TVN servoloop has a $3 \mathrm{~dB}$ attenuation at $10 \mathrm{~Hz}$ and a $45^{\circ}$ phase lag at $7 \mathrm{~Hz}$, which are characteristics in line with primary flight control actuators requirements.

An even better dynamic performance is presented by the VIGV subsystem, both when commanded by the hydraulic master servoactuator (normal operating mode), or when commanded by the backup electromechanical actuator. Figure 6 shows the VIGVs frequency response for the case of operation with the backup electromechanical actuator.

\subsection{Heat generation}

Heat is generated in the hydraulic system from hydraulic and mechanical sources. Pressure losses of the fluid flow through the hydraulic lines, passageways and metering ports, as well as the leakage flows from high to low pressure side through the hydraulic components generate heat. Windage and tare losses originated by the pumps rotation are a second source of heat generation. Most of these power losses are dependent on the system supply pressure; in particular, the losses associated to the internal leakage are almost proportional to the square of the supply pressure.

Simulations were run to assess the average heat generation during different types of aircraft missions and an average heat generation for the variable pressure system equal to $60 \%$ of that for a constant pressure system was found. This reduction of heat generation is highly significant because it greatly reduces the average temperature of the hydraulic fluid with ensuing longer life and improved system reliability.
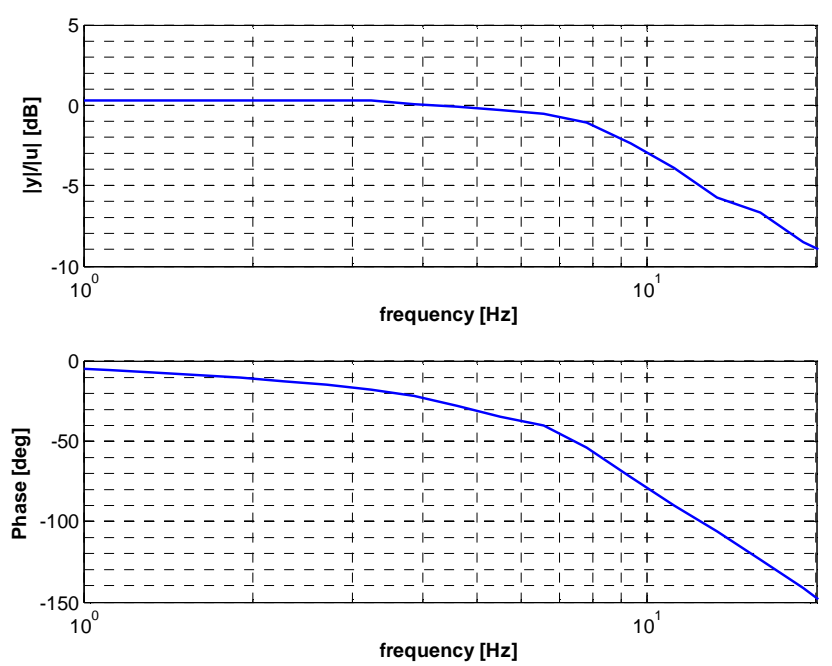

Figure 5: Nozzle servoactuators frequency response in TVN mode
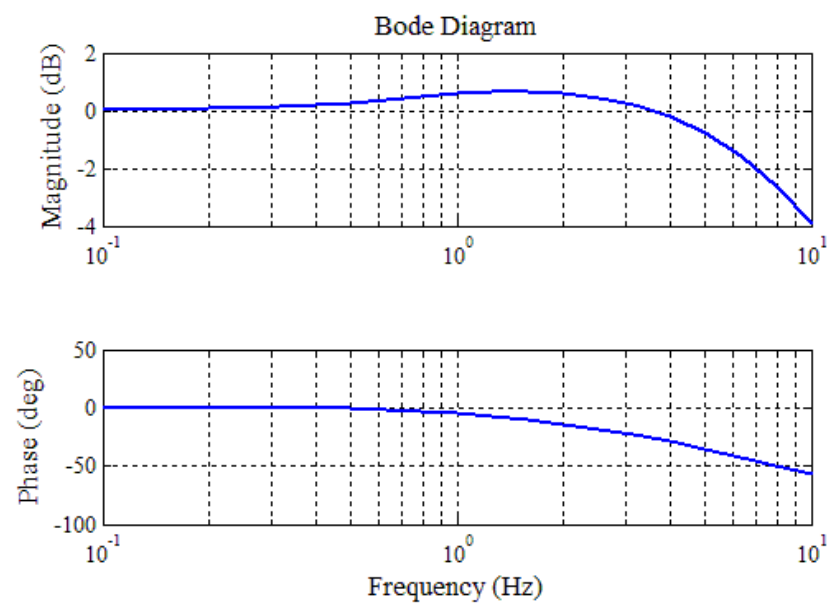

Figure 6: VIGVs frequency response when commanded by the backup electromechanical actuator

\section{Failure behaviour}

\subsection{In-flight monitoring}

The DECU uses the available information of the system status to perform a continuous monitor of the system behaviour in flight, detect failures that could impair the system performance and lead to potentially flight safety critical failures. If any of such failures is detected, the DECU takes the appropriate actions to isolate the failed component and reconfigure the system operating mode to allow a continued safe flight. The main failure cases are described hereunder. It must be emphasized once more that in case of failure ensuring the VIGVs operation is the primary objective.

\subsection{Failure of a flow control component}

All electrically operated valves and all sensors are dual electrical with the two electrical lanes interfacing with the two sections of the DECU. The two electrical lanes of the sensors are always active and the signals acquired by the 
two sections of the DECU are mutually exchanged between them, so that each DECU section has available the information coming from both sensor lanes. The electrically operated valves work in an active/standby mode; if the active section fails, the control is switched to the other DECU section.

The functionality of the electrical parts of the control valves is continuously verified by performing the current wraparound: the actually measured control current is compared with the current command. If an error is detected, the control is switched to the other electrical lane and the operation continues with full performance.

If a failure occurs of any of servovalves controlling the VEN/TVN actuators position, this is recognized as an abnormal, or uncommanded movement, or lack of movement of the VEN/TVN. In this case the solenoid operated shutoff/bypass valve is de-energized, which causes the valve to block the connection of the servovalves supply lines and to create a bypass and a connection with the return line for the actuators. In this condition, the internal pressure forces acting on the nozzle will eventually recenter the nozzle and lead it to a fail-safe position. It must be noticed that the VEN/TVN actuators, as well as the VIGV actuator, have a small bypass orifice across their control lines, which allows a slow movement under the external loads without compromising the dynamic response.

If the same type of failure occurs to the servovalve controlling the VIGV, there is no shutoff/bypass valve to isolate the VIGV servoactuator. The recovery action thus consists of totally removing the hydraulic power supply by switching off the electrically driven pump and by commanding the shutoff valve on the suction port of the engine driven pump to close. This will starve the pump and after the accumulator will be emptied by the internal leakages, there will no longer any supply pressure available. Together with these commands, the VIGV backup electromechanical actuator will be activated and will take control of the VIGV operation, while the VEN/TVN actuators will recenter as for the previous failure case. Of course, it would be possible to place a solenoid operated shutoff/bypass valve also on the VIGV supply line and allow a full system operation after a failure of the VIGV hydraulic servoactuator. However, the probability of failure of the VIGV hydraulic servoactuator is small and does not justify the additional weight, complexity and cost of another electrically operated shutoff/bypass valve.

The priority valve is a flow divider throttling the flow to the VEN/TVN actuators when necessary in order to keep full flow capability to the VIGV hydraulic actuator. It could fail in two ways: unnecessary throttling the flow to the VEN/TVN actuators, or not doing it when needed. In this second case, the VIGVs will not respond with the required dynamics and the DECU will activate the electromechanical actuator. The correct VIGV operation can thus be ensured, and appropriate troubleshooting on ground will allow to detect whether the root cause of the anomalous behaviour was a failure of the priority valve, or of the VIGV hydraulic servoactuator.

\subsection{Failure of a pump}

The engine driven pump can fail in several ways, however, all type of failures will eventually end up in:

- Lack of providing the pressurized fluid flow (could be for instance the case of fracture of the drive shaft)

- Uncontrolled flow delivery (could be the case of the pump hanger plate stuck in the maximum displacement position)

- Large internal leakage (could be the result of abnormal wear of the pump pistons)

The first of the above three failures cases is detected by a lack of pressure signaled by the relevant pressure transducer. If this failure takes place, the DECU will still provide commands to the VEN/TVN actuators, but at a greatly reduced rate to be compatible with the flow delivered by the electrically driven pump.

The second failure case will lead to an excess flow, an uncontrolled pressure increase and the consequent opening of the pressure relief valve. A large heat generation will occur very likely leading to an abnormal temperature increase. As long as the hydraulic fluid temperature remains below a safe limit, no action will be taken. When the temperature grows above the limit, the shutoff valve on the suction port is commanded to close, thereby starving the pump and the operation will continue with the electrically driven pump alone as for the previous failure case. A similar occurrence is for the third failure case of abnormally high internal leakage.

If the electrically driven pump fails, the same possible occurrences outlined above for the engine driven pump can eventually show up, though they can be originated from other causes, such as a failure of the electric motor or of its drive. Lack of providing the pressurized fluid flow will prevent the accumulator from being replenished, which could reduce to a minor degradation of the VEN/TVN servoactuators dynamic response, but leave full performance to the VIGV servoactuator. This failure is detected by a lack of pressure signaled by the relevant pressure transducer. An uncontrolled fluid flow, or an excessive internal leakage will likely remain undetected in flight because the low maximum flow rate deliverable by the electrically driven pump would probably does not entail an abnormal temperature increase for the hydraulic fluid. Should a too high temperature be actually reached, the same recovery action described for the engine driven pump failure will be taken. It could at first seem wrong to starve the engine driven pump, which is normally working, as a consequence of a failure of the electrically driven pump, but this action is effective because the internal leakage of the engine driven pump is a large source of heat generation. Therefore, bringing to zero that heat source is effective in limiting the temperature rise.

\subsection{Failure of the filter units and of the heat exchanger}

Filter units and heat exchanger are the system components contributing in maintaining a good condition of the hydraulic fluid. High pressure and low pressure filters have 
a certain dirt holding capacity; when this is exceeded the pressure drop through the filter increases rapidly, which is signalled by the relevant differential pressure transducer. Since the dirt accumulation in the filters is not a sudden event, but a progressive phenomenon, when the pressure drop grows above the acceptance limit a warning is generated by the DECU, but enough pressure differential is left for the actuators, allowing a reliable operation for the remainder of the flight. In the very remote possibility of a complete sudden clogging of the high pressure filter, VEN/TVN will not be possible, but the VIGVs could still be operated by the electromechanical actuator. Sudden filter clogging of the return filter does not impair the actuators operation because its bypass valve will open allowing direct passage of the return fluid to the heat exchanger.

It was outlined in paragraph 3.2 that the low pressure filter unit includes a flow dividing valve that bypasses part of the return flow to the heat exchanger when this flow is greater than an established limit. If this valve fails closed, then a temporary larger than normal pressure develops in the return line during rapid VEN/TVN actuators movements, which could result in a reduction of the actuation speed. No corrective action is taken by the DECU, but a warning is generated for the maintenance crew to signal the anomalous behaviour. If the valve fails open, most of the return flow will not be filtered by the low pressure filter. Although undesired, this failure normally has limited consequences because the mostly contaminated hydraulic fluid flow is the drain flow from the pumps, which does not pass through the flow dividing valve and it is routed directly to the low pressure filter. A failed open failure of the flow dividing valve can be detected by the health usage and monitoring system mentioned in section 7 of this paper.

A reduction of the heat transfer capability of the heat exchanger is very unlikely; it could occur as a result of a partial or full clogging of the heat exchanger passageways, or of an anomalous condition on the fuel side, such as a very high temperature of the cooling fuel. Whatever that failure, the end result is an abnormal temperature increase of the hydraulic fluid. The corrective action is the same as for a pump failure: firstly, close the suction line of the engine driven pump; if this is not enough, shut down the electric driven pump and operate the VIGVs with the electromechanical actuator.

\subsection{Failure of pressure and direction control components}

The pressure relief valve protecting the system from overpressures could fail in two modes: remaining closed in case of overpressures, or opening at low pressures. If the first failure mode (failed closed) occurs, this will anyhow be a second system failure: firstly there must be a component failure yielding an overpressure, and secondly the pressure relief valve failure. This combination of failures has a remote probability of occurrence, well below $1 \times 10^{-9}$ per engine operating hour because the latency period between two check of the pressure relief valve operation must be duly taken into account. It must be in fact considered that the functionality of the pressure relief valve can be periodically tested on ground by commanding a slow increase of the discharge pressure for the electrically driven pump. When the pressure corresponding to the relief valve setting has been attained, no further pressure increase should be measured because the relief valve will open. If a pressure increase above the relief valve setting is signaled by the pressure transducer, the test is immediately stopped and a relief valve failure is then signaled. However, should this combination of the above mentioned failures actually take place in flight, the pressure sensor of the failed pump which is the root cause of the excessive pressure build up will signal the abnormally high pressure and the flow from that pump will be brought to zero, either closing the pump suction (for the engine driven pump), or cutting off the electrical power supply (for the electrically driven pump).

If the pressure relief valve fails open, this will result in a decrease of the supply pressure below the normal minimum of $21 \mathrm{MPa}$ and in an increase of the temperature of the hydraulic fluid. Depending on whether the pressure relief valve fails partially or fully open two different scenarios are possible. If the pressure relief valve is only partially open, then the priority valve will adjust the control areas for the VIGVs and VEN/TVN actuators such to ensure full performance of the VIGVs. A slower than commanded movement of the VEN/TVN actuators will be detected by the DECU and an alert signal is generated. If the pressure relief valve fails fully open, then the system supply pressure falls to a very low value and no further operation is possible with the hydraulic actuators. Operation of the VIGVs is then performed by the electromechanical actuator.

The HPGU includes two check valves that can fail either closed, or open. If any of the two fails closed there could be conditions in which not enough flow can be provided to the actuators. In this case, the priority valve will limit the VEN/TVN flow and the slower than commanded actuation will be detected by the DECU, thereby generating an anomalous behaviour alert. If the check valve of the engine pump delivery line fails open, this will allow a reverse flow from the system supply line back to the engine driven pump in case of simultaneous failure of that pump. The probability of combination of these two failures in flight is extremely remote because of the low failure probability of a check valve and because the check valve functionality can be tested during periodical pre-flight checks by looking at the indication of the pressure transducer of the engine pump discharge line when only the electric driven pump is activated on ground. Anyhow, should this remote combination of failures occur in flight, VIGVs operation can be ensured by their electromechanical actuator.

If the check valve on the accumulator output line fails open, this will lead to a partial degradation of the VEN/TVN dynamic response if they are commanded during a phase of increasing system pressure because part of the fluid flow delivered by the engine driven pump will be drawn by the accumulator, hence reducing the flow available to the actuators. The functionality of this check valve can also periodically verified in pre-flight after the engine has been started and the engine driven pump is running. A high pressure level can be commanded to the pressure control 
loop of this pump, while a low pressure level is commanded to the pressure loop of the electric driven pump. If the accumulator pressure increases, that will be an indication of a failed open check valve.

\subsection{Failure of the reservoir}

The reservoir is provided with temperature and hydraulic fluid quantity transducer and with a low hydraulic fluid level switch. It also has a visual level indicator that can be used for a further check by the maintenance personnel. Aside of a structural failure, or of a seal failure that are discussed in the following paragraphs, a possible reservoir failure is the seizure of its piston that remains stuck in one position. In this case, if the system operation and the temperature variations would bring about a volume increase of the hydraulic fluid in the reservoir, such volume increase cannot be accommodated and the reservoir relief valve will first open, then the safety disc will spill the excess fluid volume overboard. This failure will eventually be detected by the health usage and monitoring system because no variation of hydraulic fluid level in the reservoir will be sensed in association with the VEN/TVN actuators movements. Those actuators are unbalanced area actuators and their movement entails a variation of the hydraulic fluid volume in the reservoir.

If the reservoir piston is stuck, but the operating conditions would bring about a decrease of the hydraulic fluid in the reservoir, there will be a decrease of the pressure at the suction ports of the pumps. This might lead, or not, to cavitation of one or both pumps and to anomalous actuators operation. If rapid variations of the actuators position are commanded, and hence large flows required, not enough flow could be available due to pumps cavitation. The priority valve will ensure the correct operation for the VIGVs actuator, but the VEN/TVN actuators will move at a slower than commanded speed. An alert signal is thus generated by the DECU, and the health usage and monitoring system will then identify the cause of the anomalous behaviour by analyzing the system response to the commands that the DECU will continue generating for the remainder portion of the flight.

\subsection{Failure of the accumulator}

A possible failure of the accumulator is the partial or total loss of the gas charge. If this happens, the accumulator function is lost, which results in a possible reduction of the actuators dynamic performance because the pumps dynamics comes into play when the actuators receive a new position command. The amount of dynamic performance reduction depends on the type of command generated by the DECU; deviations from the expected response are recognized and analyzed by the health usage and monitoring system, which provides a warning and a clue for troubleshooting to the maintenance personnel. A loss of the gas charge can be further confirmed by measuring the time necessary to rise the pressure at the system start-up when the electric driven pump is activated before the engine is started. If the gas charge is lost, there will be a noticeable reduction of the time needed to rise the pressure.
The same behaviour happens in case of a fracture of the membrane separating the gas from the liquid side of the accumulator. A possible additional consequence of this failure is the origin of an instability of the VIGVs and VEN/TVN servoactuators caused by a large quantity of gas mixed with the hydraulic fluid. If an persistent actuators position oscillation is recognized by the DECU, the solenoid operated shutoff/bypass valve of the VEN/TVN actuators is first de-energized. If the oscillation persists in the VIGVs hydraulic servoactuator, the supply pressure is removed by closing the shutoff valve on the engine driven pump suction port and stopping the electric driven pump. At the same time, the VIGVs electromechanical actuator is activated.

\subsection{Failure of the components seals and components fractures}

All hydraulic components have seals preventing internal and external leakages. A seal failure causing an internal leakage would eventually lead to more heat generation and some reduction of the maximum flow available for the actuators operation. If the additional internal leakage generated by a seal failure is small, that might remain undetected, but it will also not directly affect the system operation. However, in general a failure of an internal seal leads to a very large internal leakage with great reduction of the system capabilities and large temperature rise. For instance, if a 15 $\mathrm{mm}$ diameter cylinder seal fails and a $0.05 \mathrm{~mm}$ radial clearance is left open between piston and cylinder, a leakage flow of about $20 \mathrm{l} / \mathrm{min}$ develops for $21 \mathrm{MPa}$ pressure differential. This is a huge amount of flow that will cause a very fast temperature rise, and the same recovery actions will be taken as for the case of the failed open pressure relief valve discussed in paragraph 6.5.

If there is a failure of an external seal, this will eventually lead to a depletion of the hydraulic fluid. A rapid depletion in flight will lead to the inability of the hydraulic system to operate, but the VIGVs can still be operated by the electromechanical actuator. A slow depletion can be signalled by the health usage and monitoring system, that will generate an alert signal allowing the maintenance personnel to check the system, identify the fault and repair it before the fault grows into failure during the following flights.

The same consequences of a large external leakage are caused by a fracture of any component of the hydraulic system.

\subsection{Failure of the actuators}

The most likely failure of a hydraulic actuator is a failure of any of its seals, which has been addressed in the above paragraph 6.8. A structural failure of its cylinder will lead to a loss of hydraulic fluid, which also has been addressed in paragraph 6.8, and eventually to a mechanical disconnection. This is also the case of a fracture of the piston rod, or of the actuator eye ends. A structural failure will thus be detected by a loss of VEN/TVN operation, or of VIGVs operation. However, if there is a failure of the 
hydraulic VIGVs actuator, operation will continue under the action of the electromechanical actuator.

Failures of the servovalves controlling the actuators flows have been addressed in paragraph 6.2.

The VIGVs electromechanical actuator is used as a backup unit and its functionality is regularly checked in pre-flight to make sure that there are no dormant failures and that this actuator is thus able to ensure VIGVs operation if required to do so in flight.

\subsection{Failure of transducers and switches}

All transducers and switches are dual electrical and interface with the two sections of the DECU. The VEN/TVN and VIGVs position control loops operate in an active/standby mode, but the two electrical sections of the transducers are always active and provide the relevant signals to the two sections of the DECU that mutually exchange the informations. An individual monitoring of the transducers is continuously performed during the system operation. An effective monitoring is possible by analyzing the output signals of the individual transducers, by comparing the signals of the two sections of the same transducers, and by correlating the values of mutually related quantities.

\section{Health usage and monitoring system}

The Health Usage and Monitoring System (HUMS) has three main tasks:

- Detect failures in flight

- Check in pre-flight that all components are properly operating, including those that cannot be continuously monitored in flight

- Collect all available information and process it through dedicated algorithms to recognize possible slow degradations of the system components

If a failure is detected in flight, then the DECU takes the appropriate corrective actions to allow a continued safe flight, as outlined in the paragraphs of section 6 .

Appropriate pre-flight checks are performed before flight initiation to ensure that no failures are present, and built-intests are run to discover potentially dormant failures, such as a failure of the pressure relief valve to open. Another preflight check consists of closing and opening the motor operated shutoff valve placed on the engine driven pump suction and verify that the end position are reached, which is signaled by the closing and opening of the end-of-travel switches. If a failure is detected in pre-flight, the maintenance crew is alerted and the required maintenance action is taken.

In addition to detect failures, either in flight, or in pre-flight, thereby ensuring the system safety and preventing the occurrence of flight safety critical conditions, the DECU has available all information of the system state variables that allow the implementation of algorithms able to detect if a degradation is under way. If this happens, an alert signal is generated to warn that the system characteristics are drifting away from nominal, and that a maintenance action must be taken in due time before the fault develops into a failure.

\section{Conclusions}

The hydraulic power generation system and the associated actuators described in this paper make up a possible evolution beyond the state-of-art in the thrust control of military jet engines. The main features of the system described in the paper that provide an advancement over the existing systems are:

- Implementing a thrust vector control capability able to provide pitch and yaw control of the aircraft with dynamic performance equivalent to that of the primary flight control actuators

- Allow a safe flight condition after any single failure also for the case of a single-engine aircraft

- Improve the system maintainability by implementing an effective health usage and monitoring system capable of detecting failures, faults and degradations by performing appropriate in-flight monitoring, pre-flight checks and degradations detection. This will result in a maintainability enhancement, with an ensuing reduction of the maintenance costs and an increase of the aircraft availability. A further work is under way to fully define the health usage and monitoring system algorithms, identify the fault-to-failure mechanisms of all system components and establish the optimal values of the alert thresholds for a reliable signaling of the degradation process without generating false alarms.

\section{References}

[1] Rolls-Royce. The Jet Engine. The Technical Publication Department - Rolls-Royce plc, Derby, England, 1992. ISBN 0-902121 04-9.

[2] M. Puttre. Thrust vectoring nozzles give pilots an edge. Mechanical Engineering 115(3): 64-67, 1963.

[3] S. Ashley. 1995. Thrust Vectoring: A new angle to air superiority. Mechanical Engineering 117(1), 1995.

[4] B. Flynn, R.E. Smith, and E. Schneider. Thrust vectoring: a new dimension. Canadian Aeronautics and Space Journal, 41 (4), 1995.

[5] G.B. Gilyard, and A. Bolonkin. Optimal Pitch ThrustVector Angle and Benefits for all Flight Regimes. NASA/TM-2000-209021, 2000, available at URL http://www.nasa.gov.

[6] F.J. Lallman, J.B. Davidson, and P.C. Murphy. A Method for Integrating Thrust-Vectoring and Actuated Forebody Strakes With Conventional Aerodynamic Controls on a High-Performance Fighter Airplane. NASA/TP-1998-208464, 1998 available at URL http://www.nasa.gov. 
[7] C. Rausch, and K. Lietzau. Integrated Thrust Vectored Engine Control. Proceedings of the RTO AVT Symposium on Active Control Technology for Enhanced Performance Operational Capabilities of Military Aircraft, Land Vehicles and Sea Vehicles: 12/1-12/9, Braunscweig, Germany, May 8-11, 2000

[8] G. Jacazio, D. Ferrara, and M. Sorli. Actuation systems for the control of the engine variable inlet guide vanes: a tradeoff study. Proceedings of the AST2009 Conference on Aviation Systems Technology: 121-130, Hamburg, Germany, March 26-27, 2009

[9] G. Bardone, S. Marchetti, and A. Trovati. Actuation system for variable exhaust nozzle and inlet guide vanes on an advanced gas turbine engine. Proceedings of the RTO AVT Symposium on Design Principles and Methods for Aircraft Gas Turbine Engines 7/1 - 7/17, Toulouse, France, May 11-15, 1998

[10]G. Jacazio. Steady state and transient analysis of an adaptive fluid system. International Journal of Mechanics and Control. 3-12, Vol. 02, No. 02, 2001

[11]G. Jacazio, D. Risso, M. Sorli and L. Tomassini. Adaptive control for improved efficiency of hydraulic systems for high-speed tilting trains. Proceedings IMechE., Vol. 226 Part F: Journal of Rail and Rapid Transit; 272-283, May 2012

[12] M. Dalla Vedova, G. Jacazio, P. Maggiore and M. Sorli. Identification of Precursors of Servovalves Failures for Implementation of an Effective Prognostics. Proceedings of the 4th International Conference on Recent Advances in Aerospace Actuation Systems and Components: 111-126, Toulouse, France, May 5-7, 2010 